\title{
Surface Electronic Properties of Fe Nanoparticles on $\mathrm{c}(2 \times 2)-\mathrm{N} / \mathrm{Cu}(001)$
}

\author{
M. GetzlafF ${ }^{a}$, M. Bode ${ }^{b}$ And R. WiesendangeR ${ }^{b}$ \\ ${ }^{a}$ Institute of Applied Physics, University of Düsseldorf \\ Universitätsstr. 1, 40225 Düsseldorf, Germany \\ ${ }^{b}$ Institute of Applied Physics and Microstructure Research Center \\ University of Hamburg, Jungiusstrasse 11, 20355 Hamburg, Germany \\ We prepared nanoscaled particles consisting of ferromagnetic material \\ on a nanostructured template. This nanolithographic procedure allows to \\ fabricate high-density magnetic nanodots in a highly ordered way. For this \\ purpose, Fe particles were grown on the $\mathrm{c}(2 \times 2)-\mathrm{N} / \mathrm{Cu}(001)$ surface which \\ exhibits a checkerboard-like structure. Scanning tunneling spectroscopic mea- \\ surements demonstrate that the electronic properties of the areas with de- \\ posited material are identical to clean copper. Fe nanoparticles on the re- \\ constructed patches show a significantly different electronic behavior. These \\ observations directly hint to a covering of iron with copper on the clean \\ surface.
}

PACS numbers: 68.37.Ef, 68.55.Ac, 68.65.-k, 73.22.Dj, 81.15.Kk

\section{Introduction}

The controlled fabrication of nanometer-scaled structures is a tremendous challenge with regard to their potential applications in the design of materials with tailor-made properties. One outstanding topic in this field is the development of new high-density magnetic storage devices. Magnetic clusters are considered as one of the promising candidates [1]. For their industrially relevant production it is important to find growth modes which can combine fast growing rates and a high degree in the control of the size and shape as well as a regular arrangement.

The fabrication of nanostructured templates can be carried out in a serial way, i.e. element by element. This procedure was demonstrated by experimental techniques like electron beam lithography [2] and by scanning probe method assisted preparation [3]. These methods are not suitable for industrially relevant bulk production due to the enormous time consumption. A second approach is to 
let the nature working, i.e. to use specific interactions in a given system in such a way that self assembling on macroscopic areas occurs. Such a parallel process takes place on a significantly shorter timescale. This type of procedure is already used for a long time to prepare highly ordered layers of large organic molecules (self assembled monolayers, SAM).

There are different ways of generating nanoparticles themselves on surfaces. On the one hand, they can be prepared chemically. A skillful choice of the process parameters allows to generate particles with (nearly) identical size [4]. A self assembling with controllable distance of the clusters is realized by different ligands. A further possibility consists of self assembling of nanometer sized particles on suitable templates. One example is the faceted surface of a $\mathrm{Si}-\mathrm{Ge}$ thin film system. The evaporation of magnetic material under grazing incidence results in nanomagnets due to shadowing effects [5]. Further examples are the ordered dislocation network of an Ag film on Pt(111) [6] and the herringbone structure of the $\mathrm{Au}(111)$ surface. The deposition of Co on $\mathrm{Au}(111)$ leads to magnetic particles with dimensions in the nanometer size regime [7]. Furthermore, the nitrogen-induced reconstruction of the $\mathrm{Cu}(001)$ surface is able to serve as a nanostructured template, for the first time reported by Leibsle et al. [8]. The bombardment with high energetic nitrogen ions can be used to modify the $\mathrm{Cu}(001)$ surface in such a way that a checkerboard-like structure with typical dimensions of $5 \mathrm{~nm}$ occurs on a macroscopic scale [9-11]. A subsequent deposition of (magnetic) material allows the preparation of a highly ordered nanostructured (ferromagnetic) system [12-15].

In this paper we report on the growth and electronic properties of $\mathrm{Fe}$ clusters on the reconstructed $\mathrm{c}(2 \times 2)-\mathrm{N} / \mathrm{Cu}(001)$ surface by means of scanning tunneling microscopy and spectroscopy. Whereas the growth mode (e.g., [12]) is understood from a topographical point of view, investigations on intermixing and wetting are only rare (e.g., [16]).

\section{Experimental details}

The experiments were performed in an ultra-high vacuum (UHV) system with separate chambers for substrate preparation, sample transfer, metal vapor deposition, surface analysis, and scanning tunneling microscopy/spectroscopy (STM/STS) allowing the preparation and characterization of the sample surface without any exposure to an ambient atmosphere. The base pressure in each chamber is in the low $10^{-11}$ mbar range.

The $\mathrm{Cu}(001)$ crystal is mounted on a sample holder plate with a thickness of $1 \mathrm{~mm}$. This sample stack is prepared by numerous cycles of long-term argon ion bombardment (around $500 \mathrm{eV}$ ) at room temperature and a subsequent heating up to $750 \mathrm{~K}$. After this preparation the crystal gave a sharp $(1 \times 1)$ low-energy electron diffraction (LEED) pattern. No traces of contamination could be found by means of the Auger-electron spectroscopy (AES) and X-ray photoemission spectroscopy (XPS). 
The nitrogen-induced structures of the $\mathrm{Cu}(001)$ surface were prepared by ion bombardment of the crystal with nitrogen (purity $99.999 \%$ ) at room temperature using a beam energy of $600 \mathrm{eV}$ followed by heating to about $650 \mathrm{~K}$ for varying lengths of time. For the regular arrangement of the nitrogen-reconstructed patches one has to adjust carefully the amount of nitrogen dosage and the annealing conditions.

Fe was evaporated from a rod heated by electron bombardment. The evaporation material was degassed by prolonged heating prior to the first deposition. During Fe deposition the pressure remained below $p=8 \times 10^{-11} \mathrm{mbar}$.

All STM images shown below were measured in the constant-current mode of operation. The vertical and lateral sensitivity of the tube scanner was calibrated on monoatomic steps of the $\mathrm{Cu}(001)$ substrate and on the $(15 \times 3)$ unit cell of a carbon-induced reconstruction of the W(110) surface, respectively [17]. The STM data were plane-fitted on atomically flat terraces to correct the tilt of the sample. We enhanced the contrast of the STM images by mixing the tip height $z$ and its derivative with respect to the fast scan direction $x$, i.e. $\mathrm{d} z / \mathrm{d} x$, at different ratios of $z /(\mathrm{d} z / \mathrm{d} x)$. This image processing suggests the spectator a topography which is illuminated by an invisible light source from the left. Spectroscopic information is gained by measuring the derivative of the tunneling current $I$ with respect to the applied sample bias $U$. This so-called $\mathrm{d} I / \mathrm{d} U$ signal is a measure of the local density of states (LDOS) of the sample below the tip apex [18]. The $\mathrm{d} I / \mathrm{d} U$ signal is measured by lock-in technique. After switching off the feedback loop an ac-component $\left(U_{\bmod } \leq 20 \mathrm{mV}, \nu \approx 325 \mathrm{~Hz}\right)$ is added to the gap voltage $U$ which is ramped linearly and about 100 values of the lock-in signal are acquired. At the end of the ramp the modulation is switched off and the feedback is reactivated.

\section{Results and discussion}

The topography of a $\mathrm{Cu}(001)$ surface covered with nitrogen-induced reconstructed areas is exemplarily presented in Fig. 1a. The image shows the appearance of dark roughly square-shaped islands on this surface, i.e. these rectangular islands appear to lie deeper. These patches exhibit a random distribution for a small nitrogen coverage. Although a rumpling occurs due to the additional nitrogen atoms the averaged height does not significantly differ from that of bare copper [9]. Therefore, this observation is an electronically induced effect (see discussion of Fig. 5). Their edges are aligned along $\langle 001\rangle$ directions. After evaporation of $\mathrm{Fe}$ in the submonolayer regime the material grows as small randomly distributed islands with their edges running along $\langle 110\rangle$ directions (see Fig. 1b).

Figure 2 shows an increasing amount of activated nitrogen compared to Fig. 1. The topography presented in Fig. 2a exhibits an increased number of nitrogen-induced reconstructed areas. But an ordering occurs only on a small length scale. Even when the amount of nitrogen is very small the size of the 

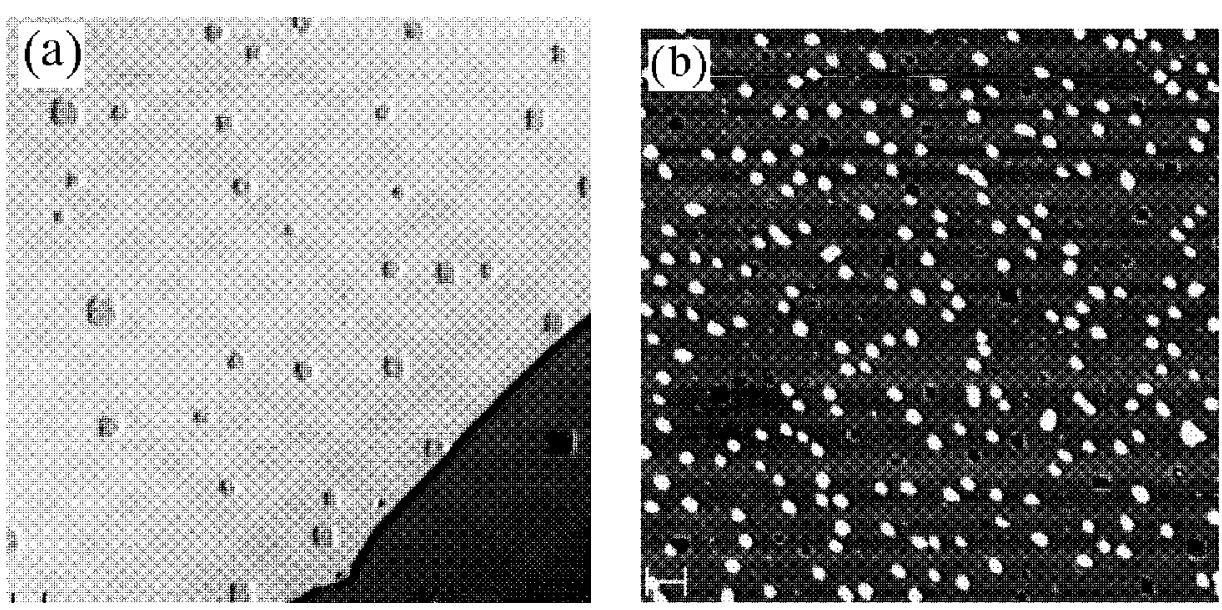

Fig. 1. $\mathrm{Cu}(001)$ surface covered with nitrogen-induced reconstructed areas. These rectangular islands appear to lie deeper which is an electronically induced effect. Their edges are aligned along $\langle 001\rangle$ directions. The scale bars correspond to $10 \mathrm{~nm}$. (a) Before iron deposition. (b) After evaporation of $\mathrm{Fe}$ in the submonolayer regime. The material is grown as small randomly distributed islands with their edges running along $\langle 110\rangle$ directions.
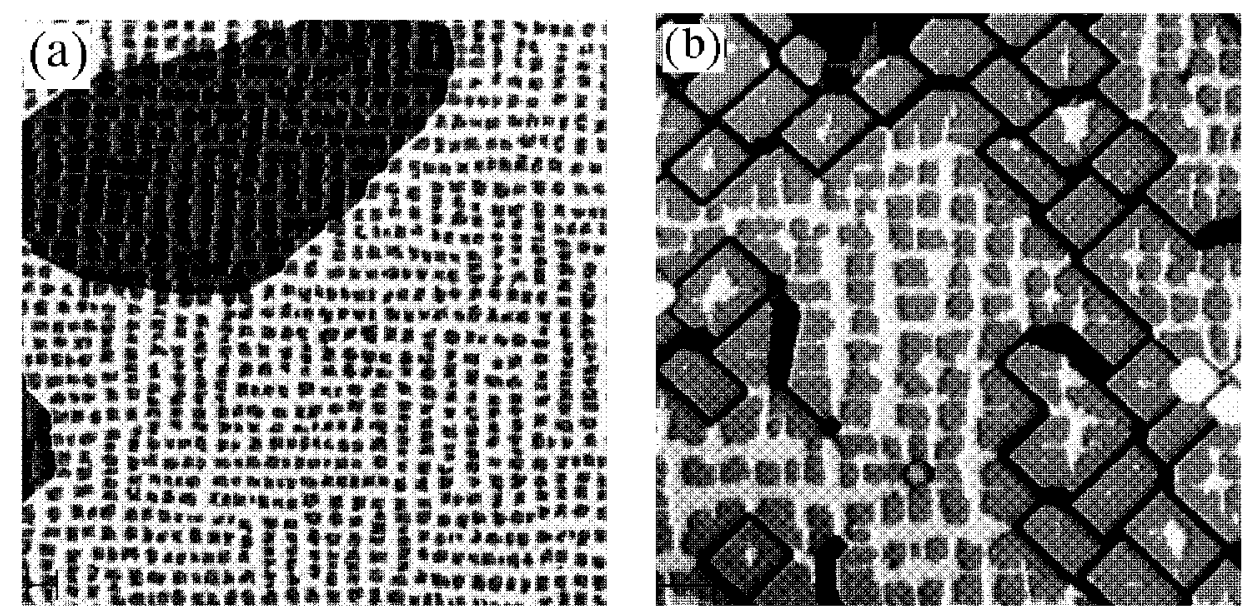

Fig. 2. Increasing amount of activated nitrogen compared to Fig. 1. The scale bars correspond to $10 \mathrm{~nm}$. (a) The number of nitrogen-induced reconstructed areas is increased. But an ordering occurs only on a small length scale. (b) Further enhancement of nitrogen ions results in a surface with square patches monoatomic height trenches.

$c(2 \times 2)$ patches is limited. During nitrogen adsorption a separation of the patches was observed [10]. An increasing size of the patches results in an introduction of two clean copper lines to split the patch into four small patches. This is believed 
to originate from reduction of the interface strain between the patch and the substrate crystal. The final total strain energy due to the lattice mismatch at the interface becomes so large that the $\mathrm{c}(2 \times 2)$ patch cannot expand further. This strain-relief mechanism is responsible for the array formation.

Figure $2 \mathrm{~b}$ demonstrates that a further enhancement of nitrogen ions results in a surface with square patches and narrow trench-like structures running in the $\langle 110\rangle$ directions. These structures are approximately $2 \mathrm{~nm}$ wide and as long as $20 \mathrm{~nm}$. In the areas between the trenches the surface exhibits a nearly perfect $c(2 \times 2)$ periodicity (not shown here). Additionally to the remaining square patches, small single atomic layer high islands occur that appear at the intersections of the trenches.
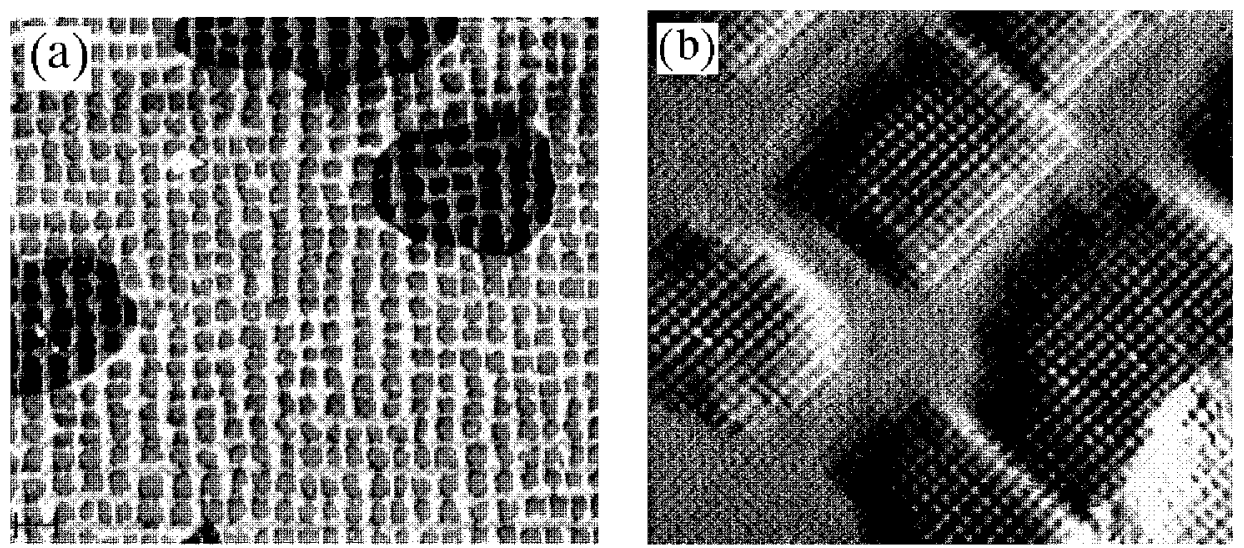

Fig. 3. STM images showing the ability of nitrogen to form self assembled highly ordered nanometer scale structures on $\mathrm{Cu}(001)$. (a) A checkerboard-like arrangement occurs on a large length scale. The scale bar corresponds to $10 \mathrm{~nm}$. (b) Atomically resolved image with a detailed view of the nitrogen-induced reconstruction. The scale bar corresponds to $1 \mathrm{~nm}$.

By adjusting the amount of nitrogen and annealing temperature $c(2 \times 2)-$ $\mathrm{N} / \mathrm{Cu}(001)$ square patches with a size of about $5 \mathrm{~nm}$ can be prepared squarely separated by a few nanometers of bare $\mathrm{Cu}(001)$. The STM images (see Fig. 3) show the checkerboard-like arrangement occurring on a large length scale and thus the ability of nitrogen to form self assembled highly ordered nanometer scale structures on $\mathrm{Cu}(001)$. This is due to the minimization of the total strain energy. A small degree of disorder remains in the width of the copper lines and the shape of the patches. The internal structure is shown in an atomically resolved image with a detailed view of the nitrogen-induced reconstruction. The atom rows within the patches are aligned along the $\langle 001\rangle$ directions. At the corners a tendency of cutting takes place thus forming octagons. 

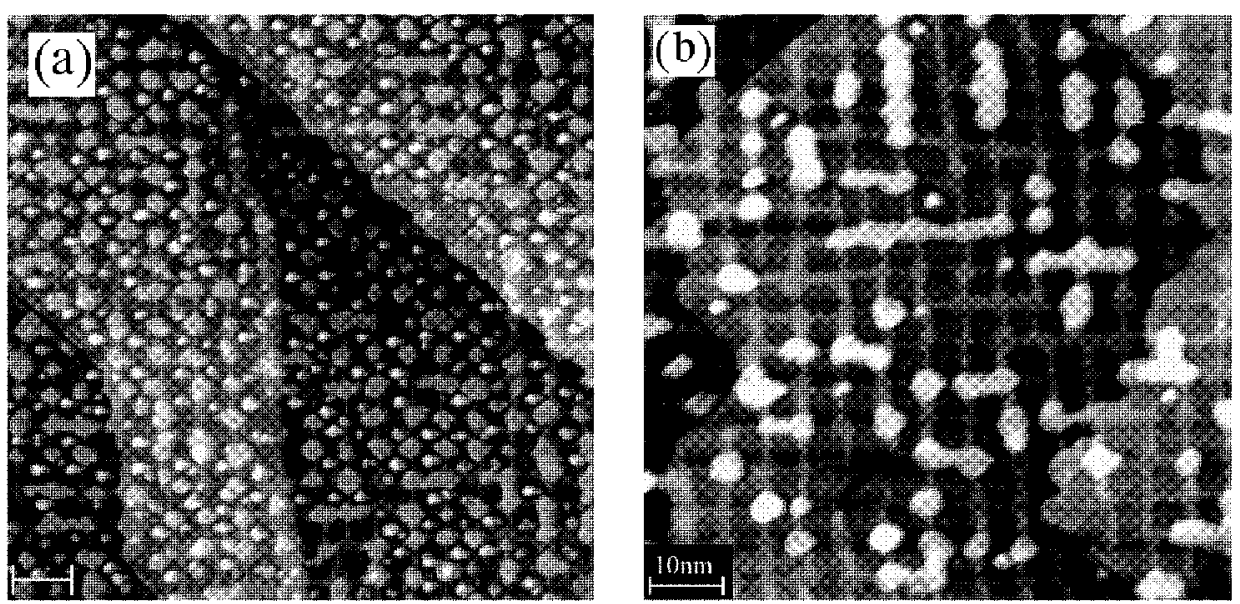

Fig. 4. Deposition of Fe on a highly ordered surface of $c(2 \times 2)-\mathrm{N} / \mathrm{Cu}(001)$ (cf. Fig. 3). (a) A larger amount of material results in $\mathrm{Fe}$ on the clean copper and additionally on the nitrogen-reconstructed areas. (b) Reducing the amount of evaporated material leads to deposition of Fe exclusively on the clean copper areas.

The effect of Fe deposition on the highly ordered surface of $c(2 \times 2)-\mathrm{N} / \mathrm{Cu}(001)$ (cf. Fig. 3) is presented in Fig. 4. Figure 4a shows a larger amount of material resulting in $\mathrm{Fe}$ on the clean copper and additionally on the nitrogen reconstructed areas. Reducing the amount of evaporated material (see Fig. 4b) leads to deposition of Fe exclusively on the clean copper areas. At the very first stage of the deposition monolayer $\mathrm{Fe}$ dots are selectively formed at the intersections of the clean copper lines and the density of the particles increase with increasing a verage thickness. After most of the intersections are covered with Fe nanodots the monolayer islands grow laterally along the clean copper lines. This change in growing characteristics is presented in Fig. 4b. As shown by Komori et al. [13] the growth

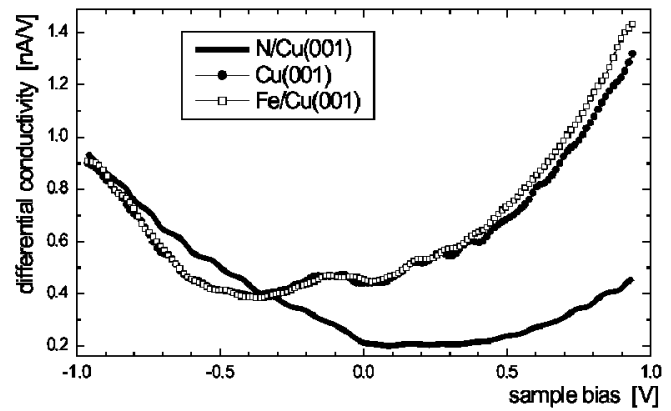

Fig. 5. Differential conductivity $\mathrm{d} I / \mathrm{d} U$ for bare copper (closed symbols), Fe on $\mathrm{Cu}(001)$ (open symbols) and the reconstructed areas $\mathrm{c}(2 \times 2)-\mathrm{N} / \mathrm{Cu}(001)$ (gray line). 
mode of Co on this nanotemplate is different. There is no selectivity between intersections and copper lines resulting in the forming of a monolayer continuous grid. Increasing amount of evaporated material results in Co islands which now grow selectively at the intersections of the wires [19]. Magnetic properties of the Fe nanodots were investigated by Finetti et al. [14]. Their data showed that the particles can be remanently magnetized parallel or perpendicular to the surface after applying a field of $0.3 \mathrm{~T}$ indicating cubic anisotropy. On the other hand, Co nanoparticles exhibit an easy axis of magnetization within the plane with an onset of sizable remanent magnetization at 1.3 monolayers [20].
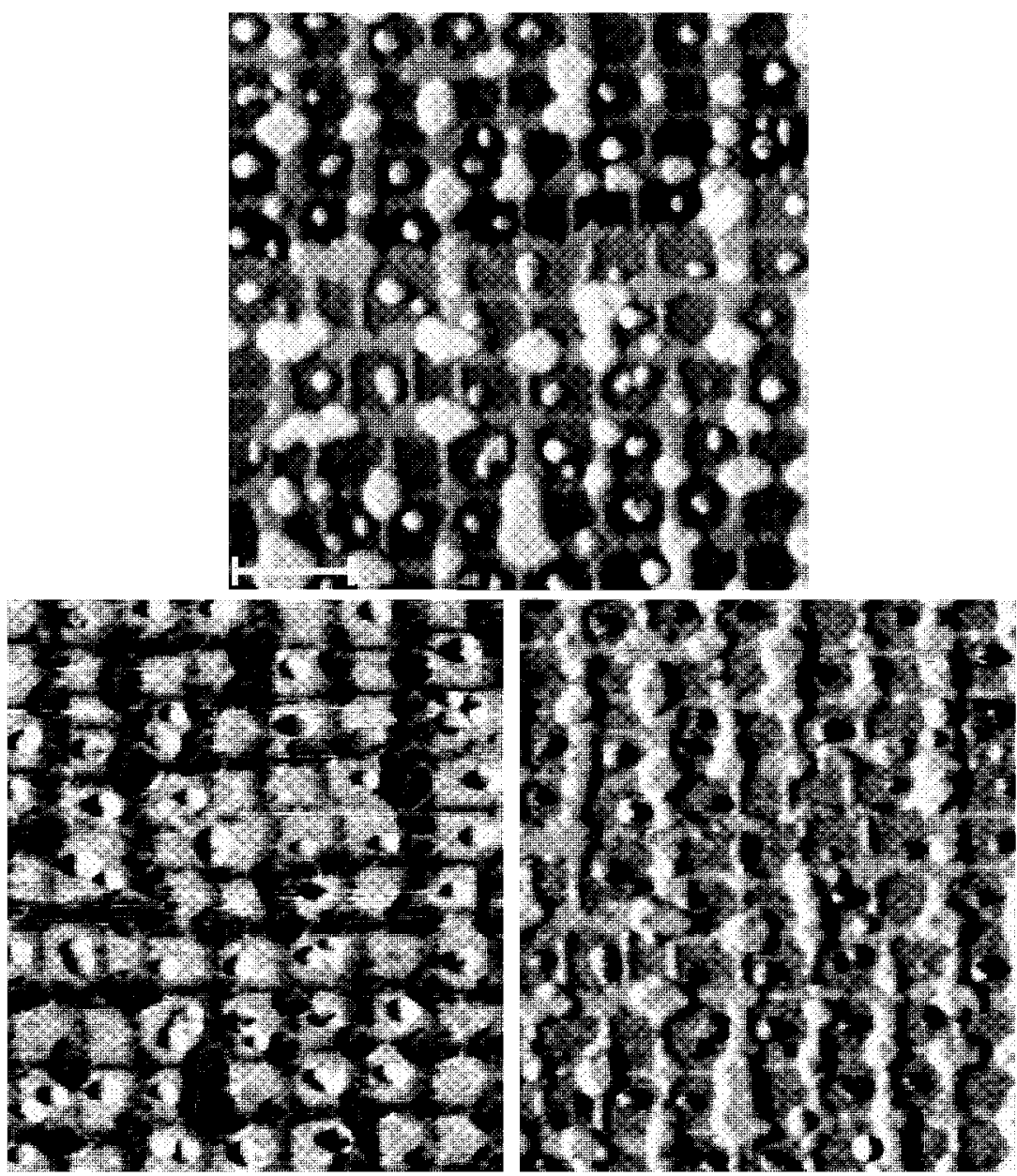

Fig. 6. Top: topographic STM image of $\mathrm{c}(2 \times 2)-\mathrm{N} / \mathrm{Cu}(001)$ after deposition of iron. The scale bar corresponds to $10 \mathrm{~nm}$. Bottom: maps of the differential conductivity at a bias voltage of $-0.5 \mathrm{~V}$ (left) and $+0.3 \mathrm{~V}$ (right). No differences in the electronic properties occur for the areas with bare copper and with deposited material. 
The electronic properties of a system presented in Fig. 4 are probed by scanning tunneling spectroscopy. The differential conductivity $\mathrm{d} I / \mathrm{d} U$ (see Fig. 5) which is a direct measure of the local density of states below the tip apex is determined for the reconstructed areas $\mathrm{c}(2 \times 2)-\mathrm{N} / \mathrm{Cu}(001)$ (gray line), the bare copper surface (closed symbols) and for the areas where deposited material is found (open symbols). It is obvious that the differential conductivity does not significantly differ for the two latter ones. In the positive sample bias regime the differential conductivity of the nitrogen-induced reconstruction is lower than for clean $\mathrm{Cu}(001)$. This results for topographic images in the constant current mode that the tip has to move towards the surface over the square patches in order to maintain the amount of tunneling electrons constant. These areas therefore appear to lie deeper (cf. Fig. 1).

The upper part of Fig. 6 shows a topographic STM image of $c(2 \times 2)-$ $\mathrm{N} / \mathrm{Cu}(001)$ after deposition of iron. Maps of the differential conductivity at a bias voltage of $-0.5 \mathrm{~V}$ (left) and $+0.3 \mathrm{~V}$ (right) are presented in the lower part. No differences in the electronic properties occur for the areas with bare copper and with deposited material. Thus, mapping of the local density of states demonstrates that this observation can be explained by the process that deposited Fe with thicknesses up to about two layers is covered with $\mathrm{Cu}$ atoms. Additionally, Fe nanoparticles on the reconstructed patches show a significantly different electronic behavior. These observations corroborate experiments by Noh et al. [21] and Kim et al. [22] which suggest that $\mathrm{Cu}$ atoms move on top of the deposited Fe if this material is located on the clean surface.

\section{Summary}

We prepared nanoscaled particles consisting of ferromagnetic material on a nanostructured template. This nanolithographic procedure allows to fabricate high-density magnetic nanodots in a highly ordered way. For this purpose, Fe particles were grown on the $\mathrm{c}(2 \times 2)-\mathrm{N} / \mathrm{Cu}(001)$ surface which exhibits a checkerboard-like structure. Scanning tunneling spectroscopic measurements demonstrate that the electronic properties of the areas with deposited material are identical to clean copper. Fe nanoparticles on the reconstructed patches show a significantly different electronic behavior. These observations directly hint to a covering of iron with copper on the clean surface.

\section{References}

[1] S. Sun, C.B. Murray, D. Weller, L. Folks, A. Moser, Science 287, 1989 (2000).

[2] S.Y. Chou, P.R. Krauss, L. Kong, J. Appl. Phys. 79, 6101 (1996).

[3] A.D. Kent, T.M. Shaw, S. von Molnar, D.D. Awschalom, Science 262, 1249 (1993). 
[4] T. Voßmeyer, G. Reck, L. Katsikas, E.T.K. Haupt, B. Schulz, H. Weller, Science 276, 1476 (1995).

[5] C. Teichert, J. Barthel, H.P. Oepen, J. Kirschner, Appl. Phys. Lett. 74, 588 (1999).

[6] H. Brune, M. Giovannini, K. Bromann, K. Kern, Nature 394, 451 (1998).

[7] B. Voigtländer, G. Meyer, N.M. Amer, Phys. Rev. B 44, 10354 (1991).

[8] F.M. Leibsle, C.F.J. Flipse, A.W. Robinson, Phys. Rev. B 47, 15865 (1993).

[9] S.M. Driver, J.T. Hoeft, M. Polcik, M. Kittel, R. Terborg, R.L. Toomes, J.H. Kang, D.P. Woodruff, J. Phys., Condens. Matter 13, L601 (2001).

[10] H. Ellmer, V. Repain, S. Rousset, B. Croset, M. Sotto, P. Zeppenfeld, Surf. Sci. 476, 95 (2001).

[11] F.M. Leibsle, S.S. Dhesi, S.D. Barrett, A.W. Robinson, Surf. Sci. 317, 309 (1994).

[12] T.M. Parker, L.K. Wilson, N.G. Condon, F.M. Leibsle, Phys. Rev. B 56, 6458 (1997).

[13] F. Komori, S. Ohno, K. Nakatsuji, J. Phys., Condens. Matter 14, 8177 (2002).

[14] P. Finetti, V.R. Dhanak, C. Binns, K.W. Edmonds, S.H. Baker, S. Daddato, J. Electron Spectrosc. Relat. Phenom. 114-116, 251 (2001).

[15] F. Komori, K.D. Lee, K. Nakatsuji, T. Timori, Y.Q. Cai, Phys. Rev. B 63, 214420 (2001).

[16] J.H. Kim, K.H. Lee, G. Yang, A.R. Koymen, A.H. Weiss, Nucl. Instrum. Methods Phys. Res. B 171, 231 (2000).

[17] M. Bode, R. Pascal, R. Wiesendanger, Surf. Sci. 344, 185 (1995).

[18] R. Wiesendanger, Scanning Probe Microscopy and Spectroscopy, Cambridge University Press, Cambridge 1994.

[19] K. Mukai, Y. Matsumoto, K. Tanaka, F. Komori, Surf. Sci. 450, 44 (2000).

[20] K.D. Lee, T. Timori, F. Komori, Surf. Sci. 454-456, 860 (2000).

[21] H.P. Noh, Y.J. Choi, J.Y. Park, I.C. Jeong, Y.D. Suh, Y. Kuk, J. Vac. Sci. Technol. $B$ 14, 1188 (1996).

[22] J.H. Kim, K.H. Lee, G. Yang, A.R. Koymen, A.H. Weiss, Appl. Surf. Sci. 173, $203(2001)$. 